\title{
Cris et chuchotements : la citoyenneté au cœur de l'enseignement de I'histoire au Québec
}

\author{
Marc-André Éthier, Jean-François Cardin \& David Lefrançois ${ }^{1}$
}

\begin{abstract}
RÉSUMÉ
Cet article propose une interprétation divergente de la controverse sur les programmes d'enseignement de l'histoire du Québec. Il soutient que les opposants au programme se trompent lorsqu'ils affirment que l'éducation à la citoyenneté est une nouveauté. Depuis le rapport Parent, l'histoire devrait familiariser les élèves avec l'attitude et la méthode des historiens, pour en faire des citoyens plus autonomes, critiques et rationnels. Les auteurs de ce texte appuient cet aspect du programme, mais déplorent le rôle de l'école dans la reproduction sociale. L'article montre que ce programme repose sur un nationalisme civique québécois, et non sur le fédéralisme, et que certains historiens et sociologues critiques du programme défendent en fait un nationalisme chauvin. Les auteurs considèrent que la lutte contre l'oppression nationale des Québécois a plus à gagner avec la formation de l'esprit critique qu'avec la mémorisation d'un récit apologétique et qu'avec l'endoctrinement, quel qu'il soit. Enfin, l'article tient ce débat non pour le signe d'une tare propre au Québec, mais pour une situation normale qui trouve son pendant ailleurs.
\end{abstract}

\section{ABSTRACT}

This article offers a different interpretation of the controversy surrounding the history curriculum in Quebec. It argues that the opponents of the current program are mistaken in claiming that its emphasis on citizenship education is a new development. Since the Parent report, the history curriculum has attempted to expose students to the approaches and methods used by historians, with the goal of making them into more autonomous, critical, and rational citizens. The authors support that aim, but deplore the role played by schools in social reproduction. They demonstrate that the current program is based around civic Québécois nationalism, and not support for federalism, and that some historians and sociologists who have criticized it are in fact proponents of a more chauvinistic nationalism. The authors believe that Quebec's struggle against national oppression will benefit more from the development of critical thinking among students than from the memorization of justificatory narratives or indoctrination of any type. Finally, rather than viewing this debate as a problem unique to Quebec, the article asserts that it has numerous precedents elsewhere in the world. 
Au milieu des années 2000, une coalition de conservateurs québécois a porté son combat sur le front éducatif. Une salve nourrie a visé le cours d'Histoire et éducation à la citoyenneté. Elle fut lancée sous le couvert de la défense de la nation québécoise contre les charges prétendument menées par le multiculturalisme, l'instrumentalisme, le postmodernisme ou l'ésotérisme ambiants. ${ }^{2}$ Cette coalition fédéra même certains progressistes livrés à sa campagne de désinformation. Nous voulons lézarder ce front hétéroclite en publiant ce texte volontairement polémique, qui cherche à rétablir les faits et à en proposer une interprétation divergente.

La première section rappelle que l'enseignement de l'histoire au Québec s'occupe depuis longtemps de la formation à la citoyenneté. La deuxième présente le débat sur la réforme et les programmes réformés. La troisième, en examinant le rapport entre les cours d'histoire actuels et le nationalisme, montre que ces cours reposent sur un nationalisme civique québécois ${ }^{3}$ et ne privilégient ni le nationalisme canadien ni l'internationalisme prolétarien. ${ }^{4}$ La dernière section analyse la position, dans ce débat, de certains professeurs d'histoire et de sociologie qui ont critiqué le programme.

\section{Les programmes d'histoire au Québec au $\mathrm{XX}^{\mathrm{e}}$ siècle}

Des intellectuels accusent les nouveaux programmes de profaner l'école en introduisant des considérations politiques impures et délétères dans ce sanctuaire jusqu'alors inviolé. ${ }^{5}$ Cette vision nostalgique est-elle juste? Nous savons depuis longtemps que la socialisation politique était omniprésente dans les écoles à travers le monde aux XIX ${ }^{\mathrm{e}}$ et XX ${ }^{\mathrm{e}}$ siècles, ${ }^{6}$ mais l'était-elle alors aussi au Québec, dans les cours d'histoire? Cette section brosse un rapide portrait de la situation prévalant avant 1960, époque du nationalisme traditionnel, puis durant la transition des années 1960-1970 et, enfin, des années 1980 jusqu’à la fin des années 1990.

Au milieu du XIX ${ }^{\mathrm{e}}$ siècle, François-Xavier Garneau a développé le prototype québécois du canon, un grand récit linéaire, fixe, téléologique et justificatif de " communautés imaginaires ", ${ }^{7}$ voire de " traditions inventées ", ${ }^{8}$ promues par "l'équipe gagnante ${ }^{9}$ pour légitimer des relations sociales asymétriques qui favorisent cette élite, quelle qu'elle soit. Durant la première moitié du $\mathrm{XX}^{\mathrm{e}}$ siècle, Lionel Groulx, historien nationaliste, l'a refondu et béni. En conséquence, tous les programmes d'études des écoles publiques québécoises fréquentées par les franco-catholiques visaient à former des citoyens vertueux. Lieu d'apprentissage du civisme, du respect des institutions et de l'autorité, ainsi que du patriotisme canadien-français, la classe d'histoire suivait le canon groulxien. La prédication catholique fortifiait tout cela.

Ainsi, en 1959, le Programme d'études des écoles élémentaires stipulait encore que l'enseignement de l'histoire devait révéler « [...] aux enfants l'action de la Providence $[\ldots] »$ et montrer que « [...] les nations n'ont de vrai bonheur» que si elles sont « [...] fidèles à la loi de Dieu ». Cet enseignement soulignait en outre « [...] l'action bienfaisante de l'Église catholique et les dévouements admirables qu'elle suscite partout où elle peut librement exercer sa mission [...] » et " [...] la pureté de nos origines canadiennes-françaises, le caractère religieux, moral, héroïque et idéaliste de nos ancêtres [...] la protection visible de la Providence sur la survivance de notre nationalisme 
[... $» .^{10}$ Par ce type de légendes, jadis présentées comme des vérités incontestables et divinement inspirées, l'on insufflait aux jeunes une fierté nationale et une mémoire collective destinée à cimenter la société.

Le souffle d'une tempête sociale emporta ce programme. En dépit des efforts des idéologues et politiciens conservateurs et des tentatives de répressions des luttes des syndicats, la pression des transformations sociales et économiques qui se précisaient dans les années 1940-1950 sema au Québec, comme aux États-Unis, en France et dans d'autres pays, un vent de changements culturels profonds qui fit se lever une tourmente sociopolitique. Des années 1960 au mitan des années 1970, ce vent balaya la province avant de commencer à tourner. L'augmentation du nombre annuel de jours de grève par personne, le niveau d'empathie qu'un nombre croissant de Québécois manifestait envers les luttes anti-impérialistes et les campagnes en faveur des droits civils à travers le monde, l'amplification et les succès des mouvements féministes et étudiants, de même que les manifestations de la transformation du nationalisme canadien-français en lutte contre l'oppression nationale des Québécois marquèrent alors un sommet. ${ }^{11}$

Ce climat turbulent poussa l'État québécois à rendre l'école obligatoire jusqu’à l'âge de 16 ans, à instaurer la gratuité des manuels scolaires et des études secondaires, à assurer aux écoles un financement plus équitable et plus satisfaisant et à s'efforcer d'unifier et de séculariser des structures et programmes auparavant ségrégués selon le genre, la foi, la langue... L'État établit un programme de prêts et bourses, alloua des subventions aux universités et contint la hausse des droits de scolarité. Cela eut pour effet d'augmenter les effectifs scolaires, le niveau d'alphabétisation, etc. ${ }^{12}$ Dans la foulée, le rapport de la Commission Parent, rendu public au milieu des années 1960, plaida pour un enseignement de l'histoire «scientifique ", destiné à former le citoyen, et attaqua durement le canon en histoire nationale. ${ }^{13}$

En 1969, le ministère de l'Éducation édicta les programmes-cadres. Ceux-ci, marqués par la psychologie humaniste, insistaient sur le développement de la personne, la créativité et la non-directivité, plutôt que sur un contenu spécifique. ${ }^{14}$ Ainsi, de 1969 à 1974, le programme d'histoire était facultatif et son contenu était adaptable à la guise des enseignants. Le programme-cadre de sciences humaines du primaire, publié en 1971, tenait en une vingtaine de pages aérées. Il recommandait quelques thèmes pour stimuler l'observation, éveiller la créativité et l'intérêt des élèves, favoriser l'établissement de liens entre les connaissances en géographie et en histoire du Québec et du monde. ${ }^{15}$ Les objectifs associés à la citoyenneté étaient beaucoup plus discrets que dans les programmes précédents ou suivants, les auteurs se bornant à affirmer qu’il fallait, au tournant du secondaire, "insister sur les éléments de civisme ». ${ }^{16}$

Cette latitude laissée aux enseignants disparut toutefois, au gré de tendances contradictoires. Ainsi, la réussite du cours d'histoire du Québec pour obtenir un diplôme d'études secondaires devint-elle obligatoire, à la suite de l'adoption, à l'Assemblée nationale, d'une motion à ce propos, déposée en novembre 1974 par le Parti Québécois. Par ailleurs, un ressac idéologique international suivit le choc pétrolier et la crise des finances publiques. ${ }^{17}$ Au Québec, au tout début des années 1980, il se traduisit par une série de mesures composites. Cela incluait des politiques de type 
thatchériste, comme des compressions budgétaires dans les services publics et des mesures affaiblissant les syndicats, dont le décret gouvernemental de 1982 fixant les conditions de travail des employés du secteur public et parapublic. Ces mesures comprenaient aussi une réforme éducative comportant tant des éléments progressistes que régressifs. Trois programmes d'histoire au contenu détaillé, libellés sous forme d'objectifs d'apprentissage mesurables et prescrits, furent publiés à partir de 1982. ${ }^{18}$ Au plan historiographique, ces programmes visaient à résorber l'écart ressenti entre l'histoire savante et le contenu de l'histoire enseignée. Dès la fin des années 1940, une poignée d'historiens québécois (Michel Brunet, Maurice Séguin, Marcel Trudel, etc.) sapaient en effet les récits encensant les actes d'individus héroïques et autres martyrs, jusque-là cautions et ressorts de l'épopée nationale, comme Dollard des Ormeaux. ${ }^{19}$ De même, en 1979, les historiens Linteau, Durocher et Robert ${ }^{20}$ publiaient le premier tome de leur Histoire du Québec contemporain-centrée sur l'évolution, non pas d'une nation, mais bien d'une société sur son territoire - perspective que les auteurs du cours d'Histoire du Québec et du Canada (quatrième secondaire) suivirent de près. Ces programmes intégrèrent donc l'étude du monde, de la période contemporaine, des mentalités, de statistiques démographiques et des structures sociales. Ils rectifièrent certains biais contre quelques groupes oubliés par le canon : Autochtones, femmes, Irlandais, Noirs, travailleurs, etc. Ils proscrivirent l'histoire strictement évènementielle, centrée sur l'empilage de faits politiques relatifs à la formation des États-nations ou à la vie des membres des élites, le plus souvent des hommes blancs.

Au plan pédagogique, la réforme devait franchir le fossé séparant l'enseignement scolaire de la recherche en institutionnalisant des pratiques qu'une majorité de chercheurs en éducation considérait comme modèles. Centrés sur l'élève, ces programmes rejetaient les coutumes didactiques visant à lui faire mémoriser un récit unique. Il s'agissait désormais d'organiser son enseignement pour faire atteindre aux élèves des objectifs d'apprentissage, de susciter l'induction, de faire manipuler des sources, de recourir aux médias audiovisuels, etc., le tout sur des contenus historiques bien ciblés et $_{\text {prescrits. }}{ }^{21}$

Le programme d'histoire nationale de $4^{\mathrm{e}}$ secondaire visait sept grandes finalités. La première était que les élèves comprennent " les principales conditions politiques, sociales, économiques, culturelles et religieuses qui ont façonné le Québec dans le contexte canadien ". La deuxième était qu'ils accroissent leurs habiletés intellectuelles et méthodologiques liées à la démarche historique. Les troisième et quatrième étaient qu'ils se sensibilisent tant "à la nécessité, aux principes et aux limites de l'analyse historique des phénomènes sociaux " qu’à la " diversité des appartenances sociales et à la réalité des solidarités et des conflits ». Les deux suivantes étaient qu'ils développent " la capacité d'analyser ses valeurs personnelles et celles de son environnement social ", de même qu'une " attitude d'ouverture et de respect à l'égard des valeurs autres que les siennes ". La dernière était de les conscientiser à leur " rôle de citoyen[s] responsable[s] de l'avenir de la collectivité $»{ }^{22}$

Avec quatre visées éducatives s'y rapportant, le programme était donc déjà explicitement axé sur l'éducation à la citoyenneté et le développement des compétences 
devant permettre d'exercer sa citoyenneté de façon éclairée en général et de formuler les problèmes identitaires en particulier :

Le programme veut tenir compte de la dimension pluraliste du passé québécois en soulignant l'apport de tous les groupes à l'histoire collective. L'histoire nationale concerne tous les Québécois, quelle que soit leur origine ethnique, linguistique, sociale ou religieuse. Par conséquent, elle doit refléter leur diversité : rendre compte des différences et les respecter, mais aussi montrer ce qui converge et ce qui est commun. La démocratie suppose l'expression de valeurs différentes, au sein d'une même société. Bien présentées, ces divergences ne peuvent que sensibiliser l'adolescent à la défense des droits fondamentaux et le conduire à plus d'ouverture à l'égard des valeurs autres que les siennes. ${ }^{23}$

Ainsi, durant plus de 20 ans, de 1984 à 2006, le cours d'histoire nationale a proposé aux enseignants et aux élèves québécois une lecture pluraliste et inclusive du passé québécois axée sur l'évolution de la société québécoise (et non de la nation québécoise, une expression absente du texte ministériel), avec comme objectif clair et explicite de former des citoyens devant fonctionner dans une société de plus en plus ouverte. ${ }^{24}$

Cependant, en dépit des attentes signifiées par les discours officiels et savants, la plupart des enseignants d'histoire relataient toujours une saga québécoise, ponctuée de faits politiques et favorisant l'identification non réfléchie à une communauté prédéfinie. ${ }^{25}$ À leur décharge, notons que, quoiqu'il s'intéressait d'abord à la compréhension de tendances socioéconomiques lourdes, le programme d'histoire nationale se structurait chronologiquement autour de ruptures constitutionnelles. En outre, l'épreuve nationale sanctionnant les études secondaires, composée de questions à choix multiples essentiellement factuels, confortait les usages liés aux questions et réponses univoques, aux exposés basés sur un schéma narratif (et actanciel) familier, comme celui du conte merveilleux, ainsi qu'aux exercices de transcription de mots et de phrases sans recherche de sens.

Certes, ce programme fut critiqué. Ainsi, Yarema Kelebay, un professeur d'histoire de l'université McGill, le rejetait au motif qu'il se basait sur l'histoire sociale et que son contenu promouvait le séparatisme et le marxisme. ${ }^{26}$ De manière générale, la "communauté " anglo-québécoise ne le trouvait pas assez canadien. Plus tard, les centrales syndicales réclamèrent à leur tour une réforme de l'école, mais en vue d'accroitre la mixité sociale, ethnique et scolaire et de mieux soutenir les milieux défavorisés pour assurer des conditions de réussite du plus grand nombre. ${ }^{27}$

Moult réflexions et discussions publiques furent menées à l'occasion des États généraux sur l'Éducation, en 1995 et 1996, et des assises du Groupe de travail sur l'enseignement de l'histoire. ${ }^{28}$ Le rapport de ce groupe, présidé par Jacques Lacoursière et dont les réflexions et recommandations inspirèrent les auteurs des actuels programmes d'histoire, ${ }^{29}$ essuya à son tour les critiques de commentateurs indépendantistes, dont la politologue Josée Legault, ${ }^{30}$ alors chroniqueuse au Devoir. Pour sa part, l'historienne Béatrice Richard accusait le rapport d'ouverture excessive au pluralisme et de rectitude politique cherchant à gommer les tensions et les conflits 
entre la majorité francophone - toujours présentée comme éternelle, monolithique et unidimensionnelle — et les autres (le Canada anglais, la minorité anglo-québécoise, les immigrants, les Autochtones, etc.). ${ }^{31}$

En somme, la formation à la citoyenneté est depuis longtemps la pierre d'assise — et d'achoppement — de l'enseignement de l'histoire au Québec.

\section{Le débat de 2006 sur l'enseignement de l'histoire nationale au Québec}

Au tout début des années 2000, le ministère de l'Éducation, du Loisir et du Sport [MÉLS] du Québec entamait le remplacement des programmes par d'autres, basés sur une approche par compétences. Au secondaire, ce processus débuta en 2005. Toujours en usage et encore en ajustement, cette réforme enchevêtre des paradigmes dits traditionnels et socioconstructivistes, un contrôle accru de l'enseignement et de l'évaluation et la confiance envers le professionnalisme des enseignants. ${ }^{32}$

Sur la base des documents et débats mentionnés plus haut, une équipe restreinte d'enseignants fut libérée de son enseignement et chargée de mener, avec l'aide d'un coordonnateur du ministère, des consultations auprès de spécialistes, historiens et didacticiens, et de rédiger le texte des nouveaux programmes d'histoire. Cette équipe faisait relire, discuter et critiquer leurs textes par une vingtaine d'autres enseignants d'histoire au secondaire, issus des différentes régions du Québec.

Les programmes d'histoire actuels consistent en deux cours devant développer trois compétences disciplinaires. Celles-ci consistent à problématiser un phénomène social, enquêter à son propos à l'aide d'outils propres aux historiens (attitudes, démarche, techniques, concepts, repères culturels et chronologiques, etc.) et prendre en compte les résultats de cette enquête dans sa vie de citoyen. Le premier cours se concentre sur l'Europe occidentale et l'Amérique du Nord, bien qu'aspirant à une histoire dite universelle. Il est enseigné la première et la deuxième année du secondaire. Le second est donné la troisième et quatrième et traite du Québec. Les deux jettent un regard comparatif sur d'autres sociétés.

Lors de la publication du premier programme, personne ne s'inquiéta de ce qu'il se structure autour du développement, par les élèves, de compétences, ni qu'il étudie des sociétés non occidentales, valorisant ainsi la diversité des sociétés, ni même qu'il rejette la mémorisation et l'encyclopédisme. ${ }^{33}$ Aussi, quand, en 2006, le personnel du MÉLS soumit à la consultation publique le second programme sur l'histoire du Québec, il ne pouvait prévoir que des militants nationalistes, identifiés à la droite comme à la gauche, ${ }^{34}$ le vilipenderaient. ${ }^{35}$

L'attaque initiale contre le projet de programme, en avril 2006, nous apparait être une réaction d'abord épidermique et peu informée d'une frange de militants nationalistes préoccupés par la propagande canadienne ${ }^{36}$ ou vexés par les rebuffades infligées à leur propre cahier d'exercices. ${ }^{37}$ L'exploitation qui s'ensuivit de la théorie du complot contre "la nation " exacerba les peurs de la majorité d'origine canadienne-française du Québec, dévoyant ainsi la conscience de l'oppression nationale. ${ }^{38}$

Sans doute pour atténuer les risques de désaveu de l'électorat francophone, le gouvernement libéral de Jean Charest revit le projet de programme au printemps et à 
l'été 2006. Les changements apportés n'accédaient pas aux demandes des opposants, mais décillaient les aveugles volontaires. Le programme restait en effet intact, outre quelques ajustements et précisions accessoires, dont le traitement de la Conquête, souligné par la création d'un chapitre à part, et l'ajout d'une série d'évènements, dont 20 pour le seul chapitre sur "L'émergence d'une société en Nouvelle-France " (comme la venue de Cartier à Gaspé en 1534 et la fondation de Québec en 1608). ${ }^{39}$ Cette version alourdie du programme fut ensuite adoptée à l'automne 2006, sans attention médiatique notable.

Débutait alors une autre phase du débat, lors de laquelle les opposants, surtout des historiens spécialistes de l'histoire du Québec, s'organisèrent mieux et tinrent un discours plus sophistiqué. Ainsi, Michel Sara-Bournet et Michel Seymour dirent ne pas vouloir restaurer une histoire "nationaliste ", mais bien défendre une " histoire nationale ", voire une histoire du national..$^{40} \mathrm{Or}$, la reprise de ce refrain par d'autres ne dupe personne : c'est bel et bien du tramage d'un canevas nationaliste traditionnel que ce chœur chante les éloges.

\section{Les programmes et le nationalisme civique}

En insistant sur l'éducation à la citoyenneté, les cours d'histoire actuels servent, selon ses opposants, de véhicule de l'unité canadienne ; ils instrumentalisent l'histoire, mise au service d'une entreprise de séduction multiculturelle canadienne. Au contraire, disent les partisans de ces programmes, insister sur l'éducation à la citoyenneté est nécessaire pour former des citoyens autonomes, critiques, éclairés et engagés. ${ }^{41}$ Qu'en est-il?

Pour la plupart, les écrits en éducation à la citoyenneté rejettent comme contreproductives les histoires aseptisées et édifiantes à l'usage de l'éducation civique ; ils les considèrent même comme incompatibles avec l'essence de l'histoire. ${ }^{42}$ Quoique l'histoire soit, pour ces auteurs, un outil ni infaillible ni même nécessaire et suffisant, ils la tiennent pour un moyen de comprendre et de critiquer la mécanique des institutions sociales. ${ }^{43}$ Pour eux, l'école doit, à partir d'une base de connaissances solide et pertinente, aider à former des citoyens éclairés et critiques et préconisent même, si elle ne défend pas des droits démocratiques et des conquêtes sociales aussi basaux que ceux-là, de renverser le système dont elle émane. ${ }^{44}$

En accord avec ces écrits, et ce, au moins depuis les années 1980, les textes ministériels québécois repoussent nommément une forme d'éducation à la citoyenneté traditionnelle s'appuyant sur la thèse de la primauté des instances collectives dans la formation de l'identité personnelle. L'approche rejetée subordonne en effet les droits et la justice sociale à la cohésion d'une " communauté " politique : "Dans le contexte de l'avènement des États-nations, il y a un peu plus d'un siècle, la généralisation de l'éducation historique dans l'école publique s'est faite à partir de préoccupations relatives à l'éducation citoyenne. Au moyen d'un récit historique, il s'agissait alors d'enseigner aux citoyens leur identité nationale ainsi que la validité de l'ordre social et politique. ${ }^{45}$ L'observance, par les élèves, de " certains rituels civiques, afin de manifester ouvertement et de réitérer leur sentiment d'appartenance à une collectivité politique spécifique ", devait favoriser cette reproduction sociale. ${ }^{46}$ 
Ces textes officiels préconisent plutôt de confier à la discipline historique « [...] la responsabilité de contribuer à former des citoyens capables d'une participation sociale ouverte et éclairée, conformément aux principes et aux valeurs démocratiques. ${ }^{47}$ Pour cela, les élèves doivent explorer et déterminer les racines historiques de leur identité sociale. ${ }^{48}$ L'histoire aiderait les élèves à découvrir leurs " fondements identitaires ", ${ }^{49}$ car, en leur « [...] donnant accès à des repères qui [leur] permettent de saisir [leur] appartenance à une collectivité qui partage des valeurs communes, notamment celles qui sont associées à la démocratie ", ${ }^{50}$ elle participe à la structuration de leur identité. Certes, les élèves doivent cultiver des valeurs prescrites, celles qui, selon le MELS, fondent la société québécoise et les disposent à exercer leur « [...] rôle de citoyen[s], dans [leur] milieu immédiat, l'école, et au sein d'une plus grande communauté ", ${ }^{51}$ mais il s'agit essentiellement de valeurs permettant à chacun de faire valoir et respecter ses opinions. L'histoire scolaire doit éduquer des « patriotes constitutionnels ", pour citer Habermas. ${ }^{52}$ Elle formerait des citoyens libres, car, en leur inculquant des valeurs démocratiques (auxquelles ils devraient adhérer pour se voir comme parties d'un contrat social, censément juste), elle les motiverait à endosser leurs fonctions de participation sociale éclairée. ${ }^{53}$ S'identifier à l'ethnie canadiennefrançaise devient alors optionnel pour s'intégrer à la nation civique québécoise et y agir dans le respect de ses institutions.

Selon Mathieu Bock-Coté et des membres de la Coalition pour l'histoire, cela pose problème : l'histoire scolaire ne doit servir ni le présent ni l'éducation à la citoyenneté, mais la seule connaissance des faits du passé, sous peine de verser dans le relativisme, le présentisme ou le marxisme. ${ }^{54}$ Pourtant, Robert Comeau et Josée Lavallée, de la Coalition, écrivaient en toutes lettres : «[n] ous croyons [...] que la connaissance de l'histoire de la société québécoise, en particulier pour les élèves issus de l'immigration, devrait être une priorité si nous voulons qu'ils puissent s'intégrer harmonieusement à leur nouvelle société d'accueil ». ${ }^{55}$ La classe d'histoire ne sert-elle pas, en construisant de l'identitaire pour ces élèves, le présent?

La plateforme de la Coalition assigne au cours d'histoire des finalités incompatibles avec le nationalisme civique dont elle se réclame pourtant. Aux dires de Robert Comeau, la "révolution pédagogique silencieuse, que le ministère a qualifiée de simple "renouveau pédagogique" et qui s'est traduite par la lutte contre la transmission des savoirs disciplinaires ainsi que par cette orientation multiculturaliste qui contredit nos politiques québécoises d'intégration des immigrants, a été mise en place sans débat démocratique. " ${ }^{56}$ L'historien Charles-Philippe Courtois s'inscrit dans cette même logique :

Ce constat est renforcé par la comparaison avec l'ancien programme. La vision amnésique de notre histoire proposée dans le nouveau programme est modelée de façon extrêmement compatible, en pratique, avec une identification au multiculturalisme canadien plutôt qu'au creuset québécois. Le Québec n'est plus une nation membre de la Confédération canadienne mais un agrégat d'identités éclatées rassemblées dans une fédération canadienne et une province caractérisée par la dualité linguistique. ${ }^{57}$ 
Puisque les nouveaux programmes entraineraient ces nouvelles dérives, ils devraient promouvoir un mode de reproduction sociale nouveau et s'éloigner des programmes précédents. Or, nous l'avons vu, les programmes de 1982 se conformaient également à toutes les dimensions standards du nationalisme civique : identification de tous les citoyens québécois à un espace civique commun, à une langue commune et à un territoire aux frontières définies.

Linterprétation que la Coalition fait des programmes ne réfléchit bien souvent qu'une image partielle, partiale, réductrice et embrouillée de leurs contenus. Ainsi, selon la relecture des coalisés, "[l]e contenu du programme Géographie, histoire et éducation à la citoyenneté [au primaire] ne retient que "des points de repère" épars $(1500,1645,1745,1820,1900,1905,1980)$ de l'histoire du Québec et du Canada qui ne correspondent pas à des moments fondateurs de l'histoire du Québec et du Canada ". ${ }^{58}$ Les élèves, insistent-ils, devraient acquérir une connaissance approfondie des « faits structurants " de l'histoire du Québec qui ont façonné l'identité collective des Québécois depuis 400 ans. L'enseignement de l'histoire ne devrait-il pas plutôt fournir aux élèves l'occasion de prendre conscience que des biais et des préjugés présentistes font que les faits du passé ne se donnent pas à eux en toute transparence et qu'il faut (par la méthode historique) chercher, aller à la source, mettre en contexte, débattre et comparer pour y voir plus clair? Quel censeur dressera la liste des connaissances qu'il serait légitime d' " acquérir ", au détriment d'autres savoirs? Quels faits faudrait-il ajouter?

Voici, en creux, la réponse des coalisés : "On ne met bien sûr pas en relief de grands personnages de notre histoire de cette époque-Champlain, Jeanne Mance, Frontenac, d'Iberville, etc ". ${ }^{59}$ Il faudrait donc les ajouter... et en enlever d'autres : "Le contenu du programme Géographie, histoire et éducation à la citoyenneté est surtout axé sur l'histoire des nations autochtones et de la diversité culturelle au Canada ". ${ }^{60}$ Pourtant, il suffit d'ouvrir le programme du primaire pour trouver, dans les contenus obligatoires, toutes les références et les figures de l'historiographie populaire (mais aucune figure amérindienne)... En effet, s'il ne propose que de grandes périodes, le programme place pourtant à l'intérieur de celles-ci des évènements bien connus de l'histoire canadienne ou québécoise, dans une rubrique intitulée "Influence de personnages et incidence d'évènements sur l'organisation sociale et territoriale " : Talon, Frontenac, Conquête, Mercier, Fédération canadienne, Révolution tranquille, Lesage, etc. On y étudie aussi les changements survenus entre 1820 et 1900, puis entre 1900 et $1980 .^{61}$

La fixation de la Coalition est en porte-à-faux avec ce qu'établit l'histoire sociale : économie, démographie, culture matérielle, pratiques quotidiennes et croyances collectives, y compris celles des femmes du peuple, ont une histoire ; les grands personnages ne sont pas les seuls véritables acteurs de changement social. Pis encore : appliquer les modifications aux programmes que propose la Coalition interfererait avec l'apprentissage de la démarche de validation, d'interprétation et de réévaluation propre à la pensée historique. Certes, le développement de la pensée historique n'est pas une priorité de la Coalition, qui plaide plutôt pour une conception conservatrice de l'enseignement du passé à l'école. 


\section{Une idéologie relevant du nationalisme conservateur}

Lors du déclenchement de l' "Affaire ", au printemps 2006, c'est autour d'une insécurité liée à la question nationale que le discours des opposants se structura. Malgré la diversité des critiques venues s'ajouter ensuite, notamment en regard des choix pédagogiques fondamentaux des nouveaux programmes (critique du constructivisme et de l'approche par compétences), cette centration nationaliste ne se démentit pas.

En 2009, Éric Bédard, Mathieu Bock-Côté, Robert Comeau, Charles-Philippe Courtois, Gilles Laporte, Laurent Lamontagne et Josée Lavallée ont fondé la Coalition pour l'histoire, avec l'appui d'un écheveau d'organismes issus du nationalisme traditionnel canadien-français, telles la Société Saint-Jean-Baptiste ou la revue L'Action nationale, donnant l'illusion d'un point de vue largement répandu. Survenant à intervalles réguliers, les coups de boutoir médiatiques de la Coalition et de rares autres opposants qui s'exprimèrent dans leur foulée s'avérèrent somme toute des feux de paille, plus ou moins limités aux pages du journal Le Devoir et à quelques émissions de la radio de Radio-Canada. Outre une poignée de didacticiens et d'historiens qui tentèrent de rectifier les demi-vérités truffant le discours des vitupérateurs, l'obstacle principal au succès de leurs offensives médiatiques a été, et reste encore, la relative indifférence populaire.

Des opposants au programme publièrent ou participèrent à la publication d'ouvrages sur l'enseignement de l'histoire, ${ }^{62}$ voulant montrer que les didacticiens n'occupent pas seuls ce champ de savoir. Par la suite, les coalisés produisirent une " enquête " ${ }^{63}$ et deux " études ", ${ }^{64}$ drapant leurs opinions et interprétations de crédibilité scientifique. La première étude porte sur la marginalisation des études québécoises et des cours d'histoire du Québec au niveau collégial. La seconde dénonce un déclin du nombre de mémoires et thèses en histoire du Québec, un désintérêt de ce thème dans les universités québécoises francophones et un manque de formation en histoire du Québec dans la formation des maitres depuis une quinzaine d'années. Malgré leur méthodologie discutable au regard des standards de la recherche universitaire, il appert qu'un argumentaire plus cohérent et relevé succéda à la méconnaissance du dossier prévalant au printemps 2006 et aux débordements de certains partisans sévissant sur les tribunes téléphoniques, forums et autres réseaux sociaux, tout en continuant de reposer sur des postulats litigieux et d'être porté par une idéologie conservatrice.

Cette critique nationaliste du programme d'histoire actuel s'articule autour de certaines idées centrales, présentées comme des "vérités ", dont nous ne rappelons ici que les plus proéminentes : ${ }^{65}$

- Les auteurs du programme cherchent à « dénationaliser » la trame du passé québécois, au profit d'une conception neutre et volontairement consensuelle de ce passé, et à gommer ainsi les conflits, surtout ceux entre la majorité francophone conquise et la minorité anglophone dominante ;

- l'éducation à la citoyenneté sert à neutraliser la question nationale et asservir l'histoire à des fins de consensus social ; 
- les idées, personnages ou évènements politiques, surtout leurs aspects constitutionnels et diplomatiques, sont dépréciés au profit d'une conception trop large, comparatiste ou matérialiste de l'analyse du passé.

Sur ce dernier point, Mathieu Bock-Côté et d'autres détracteurs du programme de 2006 condamnent en effet la conception que l'on y retrouverait d'une société québécoise devenue (dangereusement) pluraliste, ouverte et consensuelle, position illustrée par les deux citations suivantes : " J'ai eu l'occasion de l'écrire cent fois, et je ne m'y attarderai pas ici, mais après le référendum de 1995, les souverainistes ont démissionné sur le plan identitaire et se sont convertis au multiculturalisme, dont ils sont devenus les défenseurs les plus convaincus $»{ }^{66}$ " (. . .) On a remplacé les notions de " colonies » et " de métropoles » par le terme plus contemporain de "société », si bien que dans cette approche de l'histoire disparaissent les conflits intercoloniaux ". ${ }^{67}$

En somme, le programme véhiculerait une conception candide, édulcorée et jovialiste de notre passé et de notre présent, un irénisme de mauvais aloi promu par un gouvernement fédéraliste qui tenait à tout prix, pour des fins politiques, à museler le discours nationaliste militant et à maintenir la paix politique et constitutionnelle. En mars 2009, Charles-Philippe Courtois, collaborateur régulier de la revue L'Action nationale, a produit ce qui constitue peut-être l'idéaltype de la critique nationaliste contre le programme. ${ }^{68}$ Au lieu de décrire d'abord sans parti-pris l'objet de sa critique, pour mieux déployer ensuite ses arguments et interprétations, Courtois interprète d'emblée les passages expédients du programme à la lumière de ses présupposés politiques et militants. Partant, pour un lecteur impartial, impossible de connaitre l'objet de sa critique, seule celle-ci ayant droit de cité et le programme étant dès le départ caricaturé. Dans ce procédé, Courtois fait dire ce qu'il veut au programme, y compris cette idée qu’il serait une tentative de "trudeauisation » de la pensée au Québec. Il suit en cela la voie tracée par Robert Comeau et Gordon Lefebvre. ${ }^{69}$

Ces procédés ne doivent pas surprendre outre mesure. En effet, même si Courtois et la plupart des autres auteurs et propagateurs du discours critique envers le programme sont des universitaires (et s'avèrent plus mesurés quand ils diffusent des savoirs disciplinaires), ils jouent dans ce dossier un rôle d'un tout autre ordre, soit celui du militant engagé dans une cause, le nationalisme québécois conservateur. S'il s'agit de ce fait d'une action citoyenne légitime, il demeure que discourir pour militer n'est pas discourir pour comprendre. Dans la posture partisane qu'ils prennent, les enjeux sont déjà compris et les interprétations, déjà acquises et partagées au moment du fait. Nul besoin non plus d'approfondir sa connaissance du dossier, par exemple, de resituer le programme honni dans le contexte évolutif l'expliquant et montrant qu'il s'inscrit davantage dans la continuité que dans la rupture depuis le Rapport Parent. ${ }^{70}$

Par ailleurs, ce manichéisme s'autojustifie en ayant défini d'entrée le programme lui-même comme une manœuvre pour berner la population, voire comme une " attaque " contre la nation québécoise. ${ }^{71}$ Le commandement coalisé s'est donc positionné dans les médias comme les "défenseurs " face à cette menace. Il s’est posé en objecteur de conscience, porteur du "gros bon sens ", face aux machinations sournoises de "dénationalisation " ${ }^{72}$ du MÉLS — et ultimement du gouvernement Charest — aidé 
de didacticiens à l'esprit chimérique, obnubilés par leurs obsessions pédagogiques, sourds au récit explicatif de l'enseignant, en général, et du "grand roman national ", en particulier. Par conséquent, cette posture de combat autorisait les demi-vérités (" la jonction entre l'histoire et l'éducation à la citoyenneté est une nouveauté »), ${ }^{73}$ les slogans superficiels, mais efficaces au plan médiatique ("nos enfants n’apprennent plus d'où ils viennent »), ${ }^{74}$ voire de faussetés, comme celle voulant que les didacticiens mêlés au débat étaient des " concepteurs " du programme et soient stipendiés. ${ }^{75}$

Cette dernière fable est symptomatique. En effet, alors que retentissaient les cris de la Coalition, ses principaux contradicteurs furent les didacticiens, quoique leurs interventions demeuraient discrètes. Or, maints historiens de la Coalition admettent mal leur relégation dans le dossier de l'enseignement de l'histoire et l'imputent aux didacticiens. ${ }^{76} \mathrm{Si}$ le regard oblique, voire hautain, dont certains disciplinaires toisent les didacticiens ne leur est pas propre, il est davantage ancré parmi plusieurs historiens nationalistes québécois. Un des fronts de l'action de ceux-ci consiste donc à discréditer la didactique de l'histoire, associée à la pédagogie nouvelle, celle menant, selon eux, aux prétendues inepties de la Réforme. ${ }^{77}$

Ainsi, dans le numéro automne 2011- hiver 2012 de la revue Argument, un dossier intitulé «Qu'est-ce qu'être conservateur ", présenté par Éric Bédard, contient des textes d'autres dénonciateurs du programme d'histoire, dont les sociologues Mathieu Bock-Côté et Jacques Beauchemin. Ce dossier est présenté, entre autres, comme une réponse au colloque sur les néoconservateurs tenu à l'UQÀM en mars 2011. À partir d'angles d'entrée variés, les auteurs tentent de justifier le bienfondé, au Québec, d'une posture conservatrice face à la frénésie de la modernité et du changement à tout crin qui se serait emparée de la société québécoise. Ils utilisent une rhétorique parfois spécieuse, du type : "Réactive sans être nécessairement réactionnaire, la sensibilité conservatrice s'est rarement laissé encapsuler dans une idéologie ». ${ }^{78}$ Ce discours s'articule autour de quelques mantras :

- dénonciation du "progressisme " comme tendance foncièrement utopiste, propre à une certaine " gauche culturelle " trop présente au Québec, et consistant à toujours désirer le changement pour le changement $;^{79}$

- dénonciation du postmodernisme, « maladie infantile " d'une intelligentsia victime de la mode ;

- adhésion, à l'inverse, à un conservatisme identitaire et culturel, vu comme étant foncièrement pragmatique, enraciné dans le réel et réfractaire aux illusions idéologiques ;

- préservation de «nos » héritages, traditions et idéaux canadiens-français, parce qu'ils sont garants de sagesse et permettent de préserver l'unicité et la « cohésion » de « la » société québécoise contre le pluralisme débridé et bienpensant ; ${ }^{80}$ 
- dénonciation des programmes d'histoire, parce que reposant, selon eux, sur un " optimisme lyrique " annonçant une illusoire " communion fraternelle et définitive » au sein de la société, alors que l'histoire montre plutôt la nature imparfaite de l'homme, ainsi que les divisions et conflits inhérents à cette condition humaine " tragique " $;^{81}$

- dénonciation du constructivisme ruinant les savoirs disciplinaires et organisant l'école autour de marottes de didacticiens, comme l'approche par compétences (qui se substitue au récit magistral — vrai, explicatif et factuel — que l'élève devrait mémoriser pour "l'apprendre »), et ce, afin d'anéantir l'autonomie des savoirs et des enseignants. ${ }^{82}$

La droite dont il est ici question promeut un conservatisme socioculturel et identitaire plus qu'économique et couvre d'opprobre le pluralisme qui conduirait la majorité francophone d'ascendance canadienne-française à perdre ses racines et son identité nationale, ${ }^{83}$ considérée ici comme une essence, une appellation d'origine contrôlée, immuable. Mathieu Bock-Côté et d'autres reprochent surtout au Québec postréférendaire la reddition identitaire des élites nationalistes. ${ }^{84}$ Selon eux, les descendants des colons franco-catholiques doivent rejeter l'interculturalisme et le multiculturalisme. Basé sur la charte des droits et l'étatisme, soutenu par l'intelligentsia progressiste qui s'en repait, l'inter/multiculturalisme compromet le salut de cette communauté nationale majoritaire, car il en minorise l'héritage historique, sous prétexte de rédemption de ses vieux péchés xénophobes (tel l'antisémitisme durant les années 1930), pourtant déjà expiés, voire imaginaires. Il faut au contraire adhérer à une mémoire, une culture, un patrimoine historique et une identité commune et substantielle d'un sujet politique unitaire, et faire de l'assimilation de cette identité nationale la norme d'intégration des arrivants. ${ }^{85}$

Pourtant, d'autres façons de voir le débat socioidentitaire québécois existent. Deux courants (néonationalisme et fédéralisme) considèrent qu'il faut que les habitants du Québec s’identifient à une nation (québécoise ou canadienne, et non francocatholique du Québec), tandis que, pour les tenants d'un autre courant, tous les opprimés devraient embrasser la cause de la lutte contre l'oppression nationale des francophones du Canada et du Québec, car les injustices subies par certains opprimés affaibliraient les autres opprimés, alors que leur solidarité les renforcerait tous dans la lutte contre un ennemi commun. ${ }^{86}$

Autrement dit, la posture des coalisés confirme l'analyse que Martin Petitclerc ${ }^{87}$ livre d'une nouvelle génération de penseurs nationalistes, dont Éric Bédard et Mathieu Bock-Côté, qui se sont attaqués à l' " œuvre insouciante de démolition ${ }^{88}$ des historiens néonationalistes issus de la Révolution tranquille. Ils accusent ceux-ci d'avoir fait table rase du passé canadien-français pré-1960, en dénigrant l'héritage des " ancêtres " pour mieux glorifier les réformes qui se succèdent depuis la Révolution tranquille. Au néonationalisme, les historiens de la "nouvelle sensibilité ", pour reprendre l'expression de Stéphane Kelly, ${ }^{89}$ opposent un retour au nationalisme traditionnel et exigent déférence pour "nos " ancêtres dans le regard que "nous " posons 
sur «notre " passé, sur les gestes posés et les choix qu’ils ont faits. Le télescopage dans le temps, la confusion des acteurs du passé et du présent et l'essentialisation de ceuxci expliquent peut-être aussi que des rétronationalistes ne voient pas les opprimés du présent qui descendent de Français, d'Amérindiens et d'Anglais comme des alliés contre un même oppresseur.

Ce phénomène d'une droite culturelle et identitaire dénonçant des programmes d'études prônant un enseignement épistémologique de l'histoire plutôt que d'être axés sur le patriotisme et la transmission du grand roman national n'a rien de proprement québécois. Beaucoup d'autres pays débattent des politiques de la mémoire depuis les années 1980 et l'histoire scolaire forme l'un des épicentres de ces guerres d'histoire, note l'Australienne Anna Clark, dans Teaching the Nation. ${ }^{90}$

Dans presque tous les cas, le modèle s'appuie sur une attitude réactionnaire, identitaire et étroitement nationaliste menée par la droite conservatrice des pays concernés. En d'autres termes, la grille droite-gauche fonctionne bien pour interpréter les différents cas nationaux de cette réaction planétaire. En Australie, par exemple, la droite traditionaliste et identitaire attaque les récents programmes d'histoire. Cette droite, blanche et anglo-saxonne, reproche aux programmes leurs contenus trop critiques envers le colonialisme et trop ouverts au point de vue des minorités (surtout celui des Aborigènes), ainsi que le type d'enseignement méthodologique de la discipline, tout cela étant confusément perçu comme étant « de gauche ». En 2000, le premier ministre libéral John Howard (ouvertement conservateur au plan social) plaide pour un retour à la transmission des "faits " plutôt qu'un apprentissage basé sur les " débats ", dans le but avoué de générer chez les élèves une plus grande identification à la nation et un plus grand sens d'appartenance à celle-ci. ${ }^{91}$ Comme au Québec, ces "pédagogues conservateurs ", comme les appelle Clark, estiment que les jeunes ne connaissent plus les faits marquants de leur histoire, ceux qui constituent l'armature chronologicosymbolique de la nation australienne. Ils sont, au contraire, amenés à les critiquer à outrance, notamment les faits relevant du passé colonial et des relations avec les Autochtones. Il en découlerait le remplacement, chez les élèves australiens, de la fierté envers leur passé par un certain dédain de celui-ci. Certes, on aura compris que les faits dont on réclame ici la fière transmission sont les évènements liés au roman national de la majorité blanche issue de la colonisation anglaise à laquelle appartiennent les élites locales...

L'Angleterre de Margaret Thatcher subit des pressions analogues, dans les années 1980 et 1990, pour le retour des "évènements marquants " de l'histoire nationale (Henri VIII, la victoire de Nelson à Trafalgar, etc.), ${ }^{92}$ tout comme aux États-Unis, quelques années plus tard, avec la querelle des National History Standards. ${ }^{93} \mathrm{Au}$ Canada, durant les années 1990, l'attaque de Jack Granatstein ${ }^{94}$ et d'autres contre l'histoire enseignée dans les écoles (une histoire qui aurait-à tort, selon lui-capitulé sous les obus des revendications féministes, nationalistes québécoises, etc.) s'inscrit également dans les mêmes paramètres. Pour Anna Clark, les conservateurs déplorent "l'ignorance " factuelle du passé seulement lorsqu'elle concerne certains faits précis liés à la valorisation du nationalisme. Savoir « les faits » et connaitre " sa " nation deviennent des synonymes, dans une rhétorique éducative sur l'enseignement 
de l'histoire obsédée par l'évaluation de ces faits. ${ }^{95} \mathrm{Ce}$ commentaire s'applique à la situation décrite plus haut pour le Québec. ${ }^{96}$

Enfin, notons que la guerre de mémoire québécoise coïncide avec l'adoption en 2006, à l'instigation du gouverneur de la Floride, John Ellis Bush, le frère de l'exprésident George W. Bush, d'une loi sur l'éducation prévoyant que, dorénavant, « l'histoire des États-Unis doit être enseignée en tant que vraie histoire et ne doit pas adopter les points de vue révisionnistes ou postmodernes à propos de la relativité de la vérité. L'histoire américaine doit être considérée comme factuelle, et non pas comme un construit $» .{ }^{97}$ Peut-on être plus clair dans l'obscurantisme?

\section{Conclusion}

Deux idéologies reflétant des positions et des intérêts sociaux inconciliables peuvent chacune être rapprochées d'une des deux visions de l'enseignement de l'histoire s'étant heurtées dans la polémique épisodiquement relayée par les médias. Pour la Coalition, l'histoire du Québec doit servir le nationalisme québécois. Or, le récit centré sur l'identification à une nation (civique ou ethnique) socialement homogène est mal adapté pour aider les élèves à se conscientiser à la lutte des opprimés de toutes les nations contre toutes les oppressions, y compris l'oppression nationale des francophones du Canada, des Amérindiens, etc. L'histoire-récit contribue en effet au maintien des relations sociopolitiques propres à un système socioéconomique dont l'élite choisit les connaissances à " acquérir ", au détriment d'autres apprentissages, ayant pour seul mérite de consolider son pouvoir en légitimant ses connaissances. La vision de la Coalition rejoint celle des Yarema Kelebey et Jack Granatstein, à la droite du spectre idéologique. L'autre courant procède de "l'éducation nouvelle ». Il soutient que seule vaut d'être défendue une éducation propice à l'émancipation sociale, à la conscientisation, à la capacitation, à l'affirmation de l'autonomie intellectuelle et au développement de l'esprit critique. La droite y est réfractaire et l'accuse de décaler vers le rouge.

L'opposition entre ces deux courants devrait s'exprimer plus vivement avec l'accentuation de la polarisation sociale, laquelle devrait croitre à mesure que la crise économique s'aggrave. En effet, l'offensive patronale et étatique contre les droits sociaux, arrachés de haute lutte, lancée sous le prétexte de lutter pour " assainir les finances publiques ", à la suite de la crise économique de 1982, puis relancée de plus belle après celle de 1992 et la débâcle financière de 2008, s'est soldée par l'érosion, sur le front de l'éducation comme sur tant d'autres, d'une justice sociale lacunaire. Or, toutes les concessions consenties ne suffisent pas à enrayer cette crise, la crise de leur système. Il faudrait encore que les droits syndicaux et les salaires périclitent, que l'âge de la retraite soit repoussé, que le financement des services collectifs se dégrade et que plus de charges sociales échoient aux familles et individus. Pour tenter de faire avaler cet escamotage à celles et ceux qui en souffriront, l'élite déploiera un lourd arsenal idéologique défendant l'illusion d'un nous unifiant toutes les classes, ce nous qui a servi à exploiter, opprimer, désigner des boucs émissaires, justifier le racisme, la guerre et tant d'autres sacrifices. Si la diatribe contre les programmes d'histoire actuels ne 
fait certes pas paraitre mesquins les combats des Diadoques, cet effort additionnel de diversion et de couverture au service de l'assaut lancé contre la justice sociale méritait néanmoins la réplique que nous avons voulu lui opposer ici.

Nous, les auteurs de cet article, espérons que les résistances et ripostes augmenteront plus encore que les attaques conservatrices et les manipulations idéologiques. Et si l'apprentissage de l'histoire alimente l'esprit de sédition des élèves et stimule la production d'autres carrés rouges, eh! bien, soit!

\section{Notes}

1 Les auteurs, membres du Centre de recherche interuniversitaire sur la formation et la profession enseignante, (CRIFPE), sont respectivement professeurs aux universités de Montréal, Laval et du Québec en Outaouais.

2 Nicole Gagnon, "Une bonne pièce d'artillerie à placer dans l'arsenal des éducateurs et des citoyens concernés par l'avenir de nos enfants et par le sort de notre culture, " Bulletin d'histoire politique 18, 1 (2009): 229.

3 Le nationalisme civique québécois affirme qu'il existe une nation québécoise, formée des citoyens de l'actuelle province de Québec et que cette nation a le droit d'accéder à la souveraineté politique. S'ajoute en général à cela l'identification aux valeurs libérales (dont le pluralisme), aux territoire, normes et institutions publiques du Québec, ainsi qu'à la langue française. Claude Bariteau, Gérard Bouchard, Gilles Bourque, Jules Duchastel et Michel Seymour ont amplement traité de cette question. Voir aussi Denis Monière, Pour comprendre le nationalisme au Québec et ailleurs (Montréal: PUM, 2001), 12.

4 Éric Bédard, " Note au future ministre de l'éducation, " dans Contre la réforme pédagogique, Robert Comeau et Josiane Lavallée, dir. (Montréal: VLB, 2008), 119.

5 Éric Bédard et Marc Chevrier, "La transmission du patrimoine historique en péril : le Collectif pour une éducation de qualité (CEQ) se prononce, " Bulletin d'histoire politique 16, 2 (2008) ; Mathieu Bock-Côté, "Le masochisme mémoriel : considérations sur la mauvaise conscience et l'impuissance politique des sociétés occidentales, "L'Action nationale 97, 7 (2007) ; Denise Bombardier, "Les belles histoires des pays d'en haut, "Le Devoir, 29 avril 2006.

6 Voir, par exemple : Jean Anyon, "Ideology and United States history textbooks ", Harvard Educational Review ", 49 (1979); Michael W. Apple, Ideology and Curriculum (Boston: Routledge \& Kegan Paul, 1979); Michael W. Apple et Linda K. ChristianSmith, dir., The Politics of the Textbook (New York: Routledge, 1991); Lawrence Reddick, "Racial attitudes in American history textbooks of the South, " The Journal of Negro History 19, 3 (1934); Amy von Heyking, "Talking about Americans: The image of the United States in English-Canadian schools, 1900-1965, "History of Education Quarterly 46, 3 (2006): 385.

7 Benedict Anderson, Imagined Communities: Reflections on the Origin and Spread of Nationalism (Londres: Verso, 1983).

8 Eric Hobsbawm, "Inventer des traditions, " Cahiers du CERCOM, 2 (1995). Voir aussi, pour le cas de la France : L. De Cock et E. Picard, dir., La fabrique scolaire de l'histoire (Marseille: Agone, 2009).

9 Christian Laville, «Pour en finir avec la version de l'équipe gagnante, » dans Des manuels à la mémoire, ed. Henri Moniot (Berne: Peter Lang, 1984).

10 Département de l'Instruction publique, "Programme d'études des écoles élémentaires, » Département de l'Instruction publique (Québec 1959).

11 Voir Pierre Vallières, Nègres blancs d'Amérique (Montréal: Éditions Parti pris, 1967) et un de ces inspirateurs, Franz Fanon, Les damnés de la Terre (Paris: Maspero, 1961/1968). 
12 Jean-Pierre Charland, Histoire de l'éducation au Québec : de l'ombre du clocher à l'économie du savoir (Saint-Laurent: ERPI, 2005).

13 A.-M. Parent et coll., "Rapport Parent. Rapport de la Commission royale d'enquête sur l'enseignement dans la province de Québec. Deuxième partie ou Tome II (suite). Les structures pédagogiques du système scolaire. B. Les programmes d'études et les services éducatifs, " Ministère de l'Éducation du Québec (Québec 1966). Sur ce rapport, voir Jean-François Cardin, "L'histoire à l'école et l'éducation à la citoyenneté: genèse et application d'une idée ancienne qui a la vie dure, » dans Promesses et ratés de la réforme de l'éducation au Québec, dir. M. Mellouki (Québec: PUL, 2010).

14 Stéphane Martineau et Clermont Gauthier, «La réforme des programmes scolaires au Québec ", in La réforme des programmes scolaires au Québec, dir. Clermont Gauthier et Diane Saint-Jacques (Québec: PUL, 2002), 8.

15 Plusieurs jugèrent le nouveau Plan d'études en histoire du Québec et du Canada du secondaire (1970), commun à tous les élèves du Québec, francophones et anglophones, catholiques et protestants, trop " canadien ». Micheline Dumont, "L'enseignement de l'histoire d'hier à aujourd'hui, » Bulletin de liaison de la SPHQ 23, 2 (1985): 25.

16 Groupe de travail sur la réforme du curriculum, "Orientations nouvelles des sciences humaines à l'élémentaire, " Ministère de l'Éducation du Québec (Québec: Gouvernement du Québec, 1971), 21.

17 Michael W. Apple, "Creating difference: Neo-Liberalism, neo-Conservatism and the politics of educational reform, " Educational Policy, 18 (2004); Jocelyn Berthelot, Une école pour le monde, une école pour tout le monde : l'éducation québécoise dans le contexte de la mondialisation (Montréal: VLB, 2006).

18 Félix Bouvier et coll., L'histoire nationale à l'école québécoise, regards sur deux siècles d'enseignement (Québec: Septentrion, 2012).

19 Patrice Groulx, Pièges de la mémoire: Dollard des Ormeaux, les Amérindiens et nous (Hull: Vents d'Ouest, 1998); André Vachon, "Dollard des Ormeaux, Adam, » dans Dictionnaire biographique du Canada (Québec: PUL, 1966).

20 Paul-André Linteau, René Durocher, and Jean-Claude Robert, Histoire du Québec contemporain. Tome 1. De la Confédération à la crise (1867-1929) (Montréal: Boréal express, 1979).

21 Les objectifs d'apprentissage cognitif se fondaient sur la taxonomie de Benjamin Bloom (Taxonomie des objectifs pédagogiques (Montréal: PUQ, 1975)). Le néocomportementalisme (cf. Robert M. Gagné, Conditions of Learning (Toronto: HRW, 1965)) joua aussi un rôle important.

22 Ministère de l'Éducation du Québec, « Programme d'études. Histoire du Québec et du Canada. 4e secondaire. Formation générale et professionnelle " (Québec: Gouvernement du Québec, 1982), 13.

23 Ibid., 12.

24 Ibid., 11-13.

25 Michel Coron, «L'infusion de l'Éducation dans une perspective planétaire au primaire, " dans Défis et enjeux de l'Éducation dans une perspective planétaire, dir. Mohamed Hrimech et France Jutras (Sherbrooke: Éditions du CRP, 1997); Laville, «Pour en finir avec la version de l'équipe gagnante »; Yves Lenoir, "Passé, présent et futur de l'enseignement des sciences de l'humain et de la société au primaire ", dans La réforme des programmes scolaires au Québec, dir. Clermont Gauthier et Diane SaintJacques (Québec: PUL, 2002); Robert Martineau, L'histoire à l'école, matière à penser... (Montréal: L'Harmattan, 1999).

26 Yarema Gregory Kelebay, "The New Paradigm in Canadian History. Restoring reason in writing and teaching, " McGill Journal of Education 19, 3 (1984).

27 Berthelot, Une école pour le monde, une école pour tout le monde : l'éducation québécoise dans le contexte de la mondialisation.

28 Marc-André Éthier, Jean-François Cardin et Jean-Pierre Charland, «L'enseignement de l'histoire et la conscience citoyenne des élèves au Québec, " Revue LISA 3, 2 (2005). 
29 Groupe de travail sur l'enseignement de l'histoire, "Se souvenir et devenir : rapport, " Ministère de l'Éducation (Québec: Ministère de l'Éducation, 1996).

30 Josée Legault, "Histoire d'exister ", Le Devoir, 17 juillet 1996.

31 Béatrice Richard, "Se souvenir et devenir, ou oublier et disparaitre ? " Le Devoir, 24 août 1996.

32 MÉQ, "Réaffirmer l'école. Prendre le virage du succès, " (Québec: Gouvernement du Québec, 1997); « Programme de formation de l'École québécoise. Éducation préscolaire. Enseignement primaire » (Québec: Ministère de l'Éducation, 2001).

33 MELS, "Histoire et éducation à la citoyenneté. Programme de formation de l'école québécoise, secondaire, $2^{\mathrm{e}}$ cycle. Domaine de l'univers social, " (Québec: Gouvernement du Québec, 2006), 337. Les seuls à avoir manifesté leur désaccord sont Christian Rioux et Magali Fabvre ("Les manuels de l'insignifiance, " L'Actualité 33, 1 (2008)), deux ans après l'adoption du programme du premier cycle.

34 Cardin, «L'œuvre de destruction de l'identité nationale se poursuit: Quelques commentaires d'un didacticien dans la foulée des réactions au projet de programme d'histoire nationale au secondaire "; "Quebec's new history program and "la Nation": A commented description of a curriculum implementation, " dans Contemporary Public Debates Over History Education. 6th Book of the International Review of History Education Series (2010).

35 Antoine Robitaille, "Cours d'histoire épurés au secondaire. Québec songe à un enseignement " moins politique ", non national et plus " pluriel »," Le Devoir, 27 avril 2006. Denise Angers et coll., "Le programme d'histoire au secondaire. Une nouvelle version à recentrer, " Le Devoir, 28 septembre 2006. Cette lettre, publiée dans Le Devoir, est signée par 23 personnes dont une grande majorité d'universitaires.

36 Alexandre Lanoix, Historica et compagnie. L'enseignement de l'histoire au service de l'unité canadienne, 1867-2007, Histoire politique (Montréal: Lux, 2007) décrit bien cette propagande.

37 Gérald Larose, Parlons de souveraineté à l'école (Montréal: Les Intouchables, 2006).

38 Josiane Lavallée, "De l'histoire du Québec et du Canada à une histoire pour une éducation à la citoyenneté, » dans Contre la réforme pédagogique, dir. Robert Comeau et Josiane Lavallée (Montréal: VLB, 2008), 177.

39 Michel Sarra-Bournet, "Enseigner l'histoire du Québec : avec ou malgré le " renouveau pédagogique, " dans L'enseignement de l'histoire au 21 siècle au Québec, dir. Félix Bouvier et Michel Sarra-Bournet (Québec: Septentrion, 2008).

40 Sarra-Bournet, «Enseigner l'histoire du Québec : avec ou malgré le « renouveau pédagogique ", 154.

41 Walter C. Parker, "Knowing and doing in democratic citizenship education " dans Handbook of Research in Social Studies Education, dir. Linda S. Levstik et Cynthia Tyson (Mahwah: Lawrence Erlbaum, 2008), 69.

42 Marc-André Éthier, "Activités et contenus des ouvrages scolaires québécois d'histoire générale (1985-1999) relatifs aux causes de l'évolution démocratique » (Université de Montréal, 2000); William Galston, Liberal Purposes. Goods, Virtues, and Duties in the Liberal State (Cambridge: Cambridge University Press, 1991); Margaret Macmillan, The Uses and Abuses of History (Toronto: Viking Canada, 2008). Ces auteurs citent notamment M. Bloch, E.H. Carr, F. Dosse, L. Febvre, T. Holt, R. Kosseleck, H.-I. Marrou, K. Pomian, A. Prost, P. Vilar et P. Veyne.

43 Will Kymlicka, Politics in the Vernacular. Nationalism, Multiculturalism, and Citizenship (Oxford: Oxford University Press, 2001), 310.

44 Henry A. Giroux, Ideology, Culture and the Process of Schooling (Philadelphie: Temple University Press, 1981). Voir aussi John Rawls, Libéralisme politique (Paris: PUF, 1995).

45 MELS, Programme de formation de l'école québécoise, chapitre 7. Enseignement secondaire, deuxième cycle. (Québec: Gouvernement du Québec, 2006), 337.

46 Diane Lamoureux, "Droits et vertus civiques ", Bulletin de la Ligue des droits et libertés 15, 1 (1996): 15. 
47 MELS, Programme de formation de l'école québécoise, chapitre 7. Enseignement secondaire, deuxième cycle, 337-38.

48 Ibid., 341.

49 Ministère de l'Éducation, "Programme de formation de l'école québécoise. Enseignement secondaire. 1er cycle»(Québec: Gouvernement du Québec, 2004), 348.

50 Ibid., 295.

51 "Programme de formation de l'école québécoise. Enseignement secondaire. $1^{\mathrm{er}}$ cycle », 295-96.

52 Frédéric-Guillaume Dufour, Patriotisme constitutionnel et nationalisme. Sur Jürgen Habermas (Montréal: Éditions Liber, 2001).

53 MELS, «Programme de formation de l'école québécoise. Enseignement secondaire. $1^{\text {er }}$ cycle ", 338.

54 Bédard, "Note au future ministre de l'éducation ", 119; Éric Bédard et Myriam D’Arcy, Enseignement et recherche universitaires au Québec: L'histoire nationale négligée (Montréal: Fondation Lionel-Groulx, 2011), 16; Charles-Philippe Courtois, Le nouveau cours d'histoire du Québec au secondaire: l'école québécoise au service du multiculturalisme canadien? (Montréal: Institut de recherche sur le Québec, 2009), 9; Michel SarraBournet, "Quel avenir pour l'histoire du Québec?, " Bulletin d'histoire politique 15, 2 (2007).

55 Robert Comeau et Josiane Lavallée, «À quand une histoire nationale ? Pour une refonte en profondeur des programmes d'histoire, "Le Devoir, 17 octobre 2009.

56 Robert Comeau, «Il nous faut un enseignement de l'histoire nationale pour comprendre le Québec d'aujourd'hui - L'école doit aider ceux et celles qui grandissent dans une culture à y trouver leur identité, " Le Devoir, 14 juin 2009.

57 Courtois, Le nouveau cours d'histoire du Québec au secondaire: l'école québécoise au service du multiculturalisme canadien ?, 1.

58 Coalition pour la promotion de l'enseignement de l'histoire au Québec, Plateforme (Montréal: Société Saint-Jean-Baptiste de Montréal, 2009), 5.

59 Courtois, Le nouveau cours d'histoire du Québec au secondaire: l'école québécoise au service du multiculturalisme canadien?, 24.

60 Ibid., 5. Voir aussi Frédéric Bastien, «L'histoire élitiste des historiens québécois, » Le Devoir, 20 octobre 2011.

61 MÉQ, « Programme de formation de l'École québécoise. Éducation préscolaire. Enseignement primaire ", 180.

62 Robert Comeau et Josiane Lavallée, dir., Contre la réforme pédagogique (Montréal: VLB, 2008); Félix Bouvier et Michel Sarra-Bournet, dir., L'enseignement de l'histoire au 21e siècle au Québec (Québec: Septentrion, 2008).

63 Josiane Lavallée, Les résultats d'une enquête par sondage réalisée auprès des enseignants d'histoire du secondaire (Montréal: Coalition pour l'histoire, 2012).

64 Bédard et D’Arcy, Enseignement et recherche universitaires au Québec: L'histoire nationale négligée; Gilles Laporte et Myriam D’Arcy, Je ne me souviens plus. L'état désastreux de l'enseignement de l'histoire nationale dans le réseau collégial québécois (Montréal: Fondation Lionel-Groulx, 2010).

65 Vincent Boutonnet, Jean-François Cardin et Marc-André Éthier, « Les représentations quant à la place des savoirs dans l'éducation historique telles qu'elles se sont manifestées lors du débat concernant le nouveau programme d'histoire au Québec (2006-2010), » dans Actes du colloque 2011 des didactiques de l'histoire, de la géographie et de l'éducation à la citoyenneté : Que valent les apprentissages en histoire, géographie et éducation à la citoyenneté ?, INRP (Lyon: INRP, 2011); Cardin, "Histoire et l'éducation à la citoyenneté : une idée qui a la vie dure "; Éthier et Lefrançois, "Learning and teaching history in Québec: Assessment, context and outlook .»

66 Mathieu Bock-Côté, "Fragments d'une éducation conservatrice ", Arguments 14, 1 (2011): 43 . 
67 Baillargeon, «Une histoire sans mémoire. La réforme de l'enseignement de l'histoire. » Voir aussi Mathieu Bock-Côté, « Penser à l'abri du pluralisme. Genèse du multiculturalisme dans la politique contemporaine, "Controverses, 1 (2006): 142-46. Marie-Andrée Chouinard, "L'enseignement de l'histoire, pan de brouillard, " Le Devoir, 21 septembre 2009.

68 Courtois, Le nouveau cours d'histoire du Québec au secondaire: l'école québécoise au service du multiculturalisme canadien?

69 Robert Comeau et Gordon Lefebvre, "L'urgence de l'histoire ", Bulletin d'histoire politique 5, 1 (1994): 7.

70 Jean-François Cardin, « Pour un enseignement intellectuellement "riche" de l'histoire: un discours de longue date ", Canadian Diversity / Diversité canadienne 7, 1 (2009).

71 Franco Nuovo, "Drôle d'histoire ", Journal de Montréal, 28 avril 2006.

72 Mathieu Bock-Côté, La dénationalisation tranquille (Montréal: Boréal, 2007).

73 Robert Comeau, "Une histoire sans mémoire ", À bâbord!, 32 (2009).

74 Micheline Lachance, "Enseignement de l'histoire. D'une cage de homards à l'autre », Le Devoir, 13 octobre 2012.

75 Charles-Philippe Courtois, "Examen d'un programme d'histoire post-nationaliste», L'Action nationale, décembre 2007, 192.

76 Jean-François Cardin, «Les historiens et le dossier de l'enseignement de l'histoire : chronique d'un passage du centre vers la marge ", Bulletin d'histoire politique 14, 3 (2006).

77 Bédard et D'Arcy, Enseignement et recherche universitaires au Québec: L'histoire nationale négligée: 12 et passim.

78 Éric Bédard, "Présentation. Qu’est-ce qu’être conservateur ?", Argument 14, 1 (2012): 6.

79 Ibid.

80 Jacques Beauchemin, "Le conservatisme à la défense du monde commun ", Argument 14, 1 (2012).

81 Bédard, "Présentation. Qu'est-ce qu’être conservateur ?", 6.

82 Julien Prud'homme, «Réformer l'enseignement et réformer les maîtres. La transformation des programmes scolaires et ses acteurs: le cas de l'histoire nationale au Québec, 1963-2006 ", Bulletin d'histoire politique 15, 2 (2007).

83 Cela dit, certains d'entre eux déplorent "La rigidité des conventions collectives et la lourdeur bureaucratique des commissions scolaires ». Rock Beaudet et al., "Les sciences de l'éducation forment-elles de véritables maitres ?, " Le Devoir, 22 janvier 2003. Il y a donc aussi un chevauchement avec la droite économique.

84 Bock-Côté, "Fragments d'une éducation conservatrice », 43. Jacques Beauchemin, "Accueillir sans renoncer à soi-même ", Le Devoir, 22 janvier 2010; Serge Cantin, "Pour sortir de la survivance ", dans Penser la nation québécoise... dir. Michel Venne (Montréal: Québec-Amérique, 2000); Thériault, «Identité: le Manifeste de Burke. »

85 David Lefrançois, Marc-André Éthier et Stéphanie Demers, "Jalons pour une analyse des visées de formation socioidentitaire en enseignement de l'histoire ", dans Enseigner et apprendre l'histoire. Manuels, enseignants et élèves, dir. Marc-André Éthier, David Lefrançois et J.-F Cardin (Québec: Presses de l'Université Laval, 2011).

86 Ibid., 69.

87 Martin Petitclerc, « Notre maître le passé? Le projet critique de l'histoire sociale et l'émergence d'une nouvelle sensibilité historiographique, " Revue d'histoire de l'Amérique française 63, 1 (2009).

88 Ibid., 103.

89 Stéphane Kelly, Les idées mènent le Québec : essais sur une sensibilité historique (Québec: PUL, 2003). 
90 Anna Clark, Teaching the Nation. Politics and Pedagogy in Australian History (Victoria: Melbourne University Press, 2006). Voir également Gary B. Nash, Charlotte Crabtree et Ross E. Dunn, History on Trial: Culture Wars and the Teaching of the past (New York: Vintage Books, 2000), pour les États-Unis; Linda W. Rosenzweig et Thomas P. Weinland, "New directions of the history curriculum: A challenge for the 1980s ", The History Teacher, 19 (1986), pour la Grande-Bretagne; Penney Clark, "Introduction ", dans New Possibilities for the Past. Shaping History Education in Canada, dir. Penney Clark (Vancouver: UBC Press, 2011), pour le Canada; Boutonnet, Cardin et Éthier, "Les représentations quant à la place des savoirs dans l'éducation historique telles qu'elles se sont manifestées lors du débat concernant le nouveau programme d'histoire au Québec (2006-2010) ", pour le Québec; Maria Grever et Siep Stuurman, Beyond the Canon. History for the 21st Century (Basingstoke: Palgrave Macmillan, 2007); Tony Taylor et Robert Guyver, History Wars and the Classroom: Global Perspectives (Charlotte, NC: Information Age Publishers, 2011), pour d'autres pays.

91 Clark, Teaching the Nation. Politics and Pedagogy in Australian History: 1-2.

92 Jessica Balwin, "Mandated course debated ", The Sunday Telegraph, 23 septembre 1990.

93 Gary B. Nash, Charlotte Crabtree et Ross E. Dunn, History on Trial: Culture Wars and the Teaching of the past (New York: Vintage Books, 2000).

94 Jack Granatstein, Who killed Canadian history? (Toronto: Harper Collins, 1998).

95 Clark, Teaching the Nation. Politics and Pedagogy in Australian History.

96 Il s'applique également à l'histoire ethnocentrique (afroaméricaine, en l'occurrence) véhiculée par la Nation d'Islam ou d'autres associations de classe moyenne dont les visions racistes divisent les opprimés. Greg Rosenberg et Sam Manuel, « Million Man March' Leaders Offer No Way Forward ", The Militant 59, 37 (1995).

97 Jonathan Zimmerman, "Revisionists, get out of Florida ", Los Angeles Times, 7 juin 2006. 